University of Nebraska - Lincoln

DigitalCommons@University of Nebraska - Lincoln

2009

\title{
The surface relaxation and band structure of $\mathrm{Mo}(112)$
}

\author{
Ning Wu \\ University of Nebraska-Lincoln \\ Yaroslav B. Losovyj \\ University of Nebraska-Lincoln, ylozovyy@indiana.edu \\ Zhaoxian Yu \\ Zhongshan University \\ Renat F. Sabirianov \\ University of Nebraska at Omaha, rsabirianov@mail.unomaha.edu \\ Wai-Ning Mei \\ University of Nebraska at Omaha, physmei@unomaha.edu \\ See next page for additional authors
}

Follow this and additional works at: https://digitalcommons.unl.edu/physicsdowben

Part of the Physics Commons

Wu, Ning; Losovyj, Yaroslav B.; Yu, Zhaoxian; Sabirianov, Renat F.; Mei, Wai-Ning; Lozova, N.; Colón Santana, J. A.; and Dowben, Peter A., "The surface relaxation and band structure of $\mathrm{Mo}(112)$ " (2009). Peter Dowben Publications. 244.

https://digitalcommons.unl.edu/physicsdowben/244

This Article is brought to you for free and open access by the Research Papers in Physics and Astronomy at DigitalCommons@University of Nebraska - Lincoln. It has been accepted for inclusion in Peter Dowben Publications by an authorized administrator of DigitalCommons@University of Nebraska - Lincoln. 


\section{Authors}

Ning Wu, Yaroslav B. Losovyj, Zhaoxian Yu, Renat F. Sabirianov, Wai-Ning Mei, N. Lozova, J. A. Colón

Santana, and Peter A. Dowben 


\title{
The surface relaxation and band structure of $\operatorname{Mo}(112)$
}

\author{
Ning Wu ${ }^{1}$, Ya B Losovyj ${ }^{1,2}$, Zhaoxian Yu $^{3}$, R F Sabirianov ${ }^{4}$, \\ W N Mei ${ }^{4}$, N Lozova ${ }^{2}$, J A Colón Santana ${ }^{1}$ and P A Dowben ${ }^{1,5}$ \\ ${ }^{1}$ Department of Physics and Astronomy and the Nebraska Center for Materials and \\ Nanoscience, University of Nebraska-Lincoln, Lincoln, NE 68588-0111, USA \\ ${ }^{2}$ The J Bennett Johnston Sr. Center for Advanced Microstructures and Devices, \\ Louisiana State University, 6980 Jefferson Highway, Baton Rouge, LA 70806, USA \\ ${ }^{3}$ School of Physics and Engineering, Zhongshan University, Guangzhou 510275, \\ People's Republic of China \\ ${ }^{4}$ Department of Physics, University of Nebraska at Omaha, Omaha, NE 68182-0266, USA \\ E-mail: pdowben@unl.edu
}

Received 4 May 2009

Published 5 November 2009

Online at stacks.iop.org/JPhysCM/21/474222

\begin{abstract}
The experimental and theoretical surface band structures of Mo(112) are compared. This surface band structure mapping is presented with corrections included for the lattice relaxation of the Mo(112) surface. Quantitative low energy electron diffraction (LEED) has been used to determine the details of the Mo(112) surface structure. The first layer contraction is $14.9 \%$ by LEED intensity versus voltage analysis and is in general agreement with the $17.6 \%$ contraction found from total surface energy optimization. The electronic band structure is mapped out along $\bar{\Gamma}-\bar{X}$ and $\bar{\Gamma}-\bar{Y}$ of the surface Brillouin zone (SBZ). There is strong evidence of electron-phonon coupling particularly in the region of the Fermi level band crossing at $0.54 \AA^{-1}$.
\end{abstract}

\section{Introduction}

Angle-resolved photoemission, in principle, measures the wavevector dependent quasi-particle spectra function [1-3]. The surface structure and changes to the surface structure due to surface lattice relaxation and surface reconstructions will also alter the surface band structure. To obtain a complete picture of the band structure of a metallic surface, both electron-phonon interactions and the details of the surface lattice structure must be considered. As an example of this interplay of the quasi-particle band structure and surface lattice structure, we review the surface band structure of Mo(112).

Molybdenum is an excellent example for illustrating this interplay between structure and electron-phonon coupling. Although surface states and the associated discussion of the existence of surface states and surface resonances has a very long history [4-9], in fact the first surface states shown to exist on metal single crystal surfaces were found for tungsten [10] and molybdenum $[11,12]$. Some surfaces of molybdenum, in

5 Address for correspondence: Department of Physics and Astronomy and the Nebraska Center for Material and Nanoscience, Behlen Laboratory of Physics, University of Nebraska, Lincoln, NE 68588-0111, USA. particular the (100) surface [13-22], are well known for surface reconstructions driven by a Peierls' like instability [23-26] that results from a charge density wave transition coupling with the surface states at the Fermi level. In brief, the spanning of the nested portions of the two-dimensional Fermi surface by the surface phonon wavevector results in the periodic lattice displacement, opening up an energy gap at the Fermi level. This view is not, however, entirely supported by experiment, as in some cases the $k$-vector matching does not fit expectations for the W(100) and Mo(100) surfaces [27, 28].

Inglesfield [22] argues persuasively that the atoms move in an anharmonic potential and thus the phonon frequencies are temperature dependent. This latter view by Inglesfield [22] means that details of the surface structure, surface charge density perturbations that couple to the surface states and the quasi-particle band structure of a surface state in the vicinity of the Fermi level all play a role in establishing not only the surface electronic structure, but the surface structure stability. The surface states that cross the Fermi level are indeed responsible for the surface relaxation and reconstruction but it is the combination of perhaps a more conventional charge density wave mechanism and strong electron-phonon coupling 
that may drive the surface reconstructions that occur at some molybdenum surfaces.

Admittedly, the investigation of the surfaces of molybdenum has a long history, as just indicated. Even at this stage, a review of what we know of these surfaces deserves some re-evaluation given that for the Mo(112) surface, we can now bring together a much more unified picture of the interplay between the surface relaxation and surface band structure, while at the same time illustrating the electron-phonon coupling mass enhancement that occurs for the surface state of $\mathrm{Mo}(112)$ in the vicinity of the Fermi level. This review of the electron-phonon coupling that occurs at the surface of Mo(112) seems particularly fitting as one of the first surfaces, for which the electron-phonon coupling (quasi-particle) mass enhancement was observed, was also a surface of molybdenum: $\operatorname{Mo}(110)$ [3].

\section{Experimental details}

While, as we have indicated, the study of the Mo(112) surface structure and electronic structure has a rich and long history, we have re-evaluated some of the key parameters with new measurements. In addition, we have also tried to provide a more complete picture of the $\operatorname{Mo}(112)$ surface with new high resolution photoemission measurements.

The Mo(112) surfaces were prepared by using standard methods of flashing and annealing in oxygen [29-39]. The low energy electron diffraction (LEED) experiments were taken in the ultrahigh vacuum (UHV) chamber with a pressure of $1 \times$ $10^{-10}$ Torr to establish the surface relaxation of the $\operatorname{Mo}(112)$ surface combined with photoemission. The LEED established that the surface is free of contamination.

As a part of the surface structural characterization, the LEED intensity of the diffracted beams was recorded as a function of the incident electron kinetic energies (ranging from 110 to $350 \mathrm{eV}$ ). All the data were collected at normal incidence and the LEED intensities of 14 beams were obtained as a function of kinetic energy.

Angle-resolved photoemission spectra along the chain direction and perpendicular to the chain were obtained using plane polarized synchrotron light dispersed by a $3 \mathrm{~m}$ toroidal grating monochromator [29-31, 40, 41], at the Center for Microstructures and Devices (CAMD) [42]. The measurements were made in an ultrahigh vacuum (UHV) chamber employing a hemispherical electron analyzer with an angular acceptance of $\pm 1^{\circ}$, as described elsewhere [29-31, 40, 41]. The combined resolution of the electron energy analyzer and monochromator is $120-150 \mathrm{meV}$ for the higher photon energies $(50-120 \mathrm{eV})$, but higher resolution (about $80 \mathrm{meV}$ ) was obtained at lower photon energies of 15-40 eV. The photoemission experiments were undertaken with a light incidence angle of $45^{\circ}$ with respect to the surface normal. All binding energies are referenced to the Fermi level.

The high resolution photoemission studies were carried out on the $3 \mathrm{~m}$ normal incidence monochromator (NIM) beamline that consists of a water cooled ellipsoidal entrance mirror with a $70 \mathrm{mrad}$ acceptance angle of horizontal radiation from a dipole magnet at CAMD [43], as described elsewhere [44]. The normal incidence monochromator is combined with an angle-resolved ultraviolet photoemission (ARUPS) endstation, which consists of a magnetic field shielded ultrahigh vacuum (UHV) chamber equipped with the electron energy analyzer (Scienta SES200 electron energy analyzer) [43]. Gas phase resolution tests as well as tests of resolution based on the gold (111) surface state show that the combined resolution (using He I radiation) is $9 \mathrm{meV}$ or less at the lowest possible temperature we have obtained. The measured Fermi edge broadening of gold films at about of $30 \mathrm{~K}$ is less than $15 \mathrm{meV}$ representing an upper bound to the combined resolution of the beamline and the electron energy analyzer [43]. The high resolution photoemission spectra presented here are for a photon energy of $18 \mathrm{eV}$, resulting in an improved wavevector resolution because of the lower photon energy.

To include the experimental band mapping of the unoccupied states for $\operatorname{Mo}(112)$, we also revisit prior inverse photoemission results [30-32] as a part of our overview of the Mo(112) surface electronic structure. These measurements are detailed elsewhere [30-32].

\section{Determination of the Mo(112) surface structure}

Low energy electron diffraction (LEED) intensity versus electron kinetic energy data, when complemented by dynamical scattering calculations, i.e. $I(V)$ analysis, is a useful tool to study the surface structure, especially relaxation and reconstruction. For the Mo(112) surface, the surface adopts a ridge and trough structure: a furrowed surface schematically illustrated in figure 1(a). Ideally, Mo atoms in the top layer form close packed rows along the [11 1 ] direction, with a spacing of $4.45 \AA$ between them in the [110] direction [30-32, 45-47]. This view of the Mo(112) surface is consistent with scanning tunneling microscopy studies [35, 36]. Figure 1(b) shows the LEED pattern at an electron energy of $220 \mathrm{eV}$ at room temperature, and no superstructure spots are observed over a wide range of temperatures, so for the measurements reported here, the clean $1 \times 1$ structure of $\operatorname{Mo}(112)$ applies without the complication of an in-plane surface reconstruction. Surface layer relaxation does occur and must be considered, as discussed below.

The surface structure determination was performed by LEED, by model fitting to the measured diffracted LEED beam intensities recorded as a function of the incident electron kinetic energies (ranging from 110 to $350 \mathrm{eV}$ ), and a selection of such $I(V)$ curves have been plotted in figure 2 . The modeling of the experimental data used the automated tensor LEED (TLEED) method [48]. In the TLEED method, a full dynamical calculation is carried out only for a reference structure, and approximate $I(V)$ spectra were then obtained for nearby structures using easily calculated tensors. This program also employs a directed search algorithm, which can rapidly converge to a minimum of the $R$-factor with respect to small displacements from the reference structure. As a result, the automated TLEED method is extremely fast and can locate a nearby local minimum in seconds, even for very complicated structures. In our calculations, the scattering phase shifts of Mo 


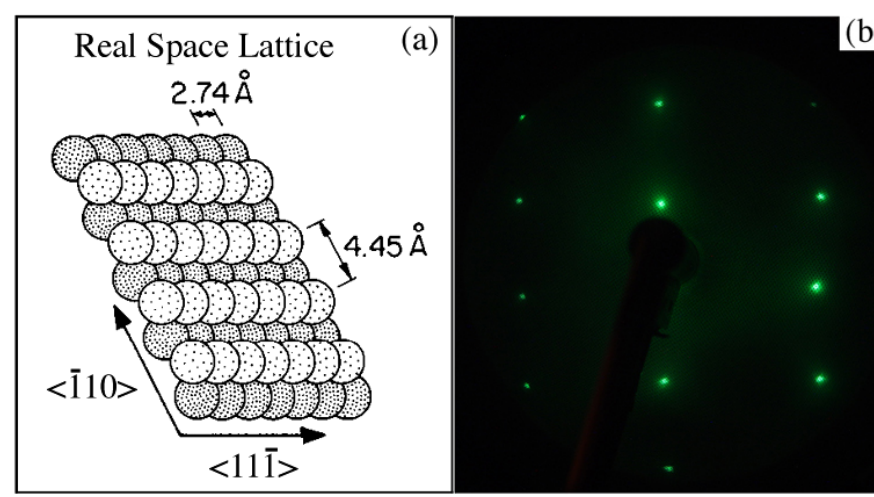

(c)

Surface Brillouin Zone

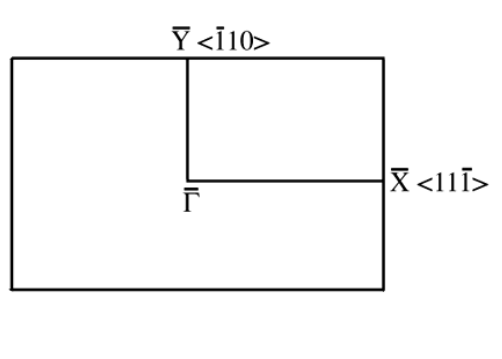

Figure 1. A schematic diagram of the rectangular surface structure of unreconstructed $\mathrm{Mo}(112)$ (a) and the corresponding LEED pattern taken at an incident electron energy of $220 \mathrm{eV}$ (b). A schematic diagram of the surface Brillouin zone for the Mo(112) is illustrated, with the critical points labeled following the common convention (c).

(This figure is in colour only in the electronic version)

atoms were generated with the Barbieri-Van Hove phase shift package. Up to 10 phase shifts were used in the calculations. The Debye temperature for Mo was set to $450 \mathrm{~K}$. The energy independent real and imaginary parts of the inner potential $\left(V_{\mathrm{r}}\right.$, $V_{\mathrm{i}}$ ) were set to $8.0 \mathrm{eV}$ and $-5.0 \mathrm{eV}$, respectively. These nonstructural parameters were fixed at the initial stage of analysis and, together with structural parameters, were optimized in the final refinement of the favored structures.

We began our search for a model that would best fit the experimental LEED $I(V)$ data with variations of structural parameters of the first six layers, starting from their bulktruncated positions. In the initial stage, all non-structural parameters were kept unchanged. The best structure so found had an $R$-factor of 0.28 . In the final step of refinements, both the structural parameters and the non-structural parameters (i.e. the Debye temperature and the inner potential) were optimized. As expected, this further optimization step does not lead to a significant improvement of the $R$-factor, yielding a final structure with an $R$-factor of 0.27 . Figure 2 shows the LEED $I(V)$ experimental spectra for the clean Mo(112) $(1 \times 1)$ surface, together with the best-fit calculated spectra and there is good agreement between experiment and theory.

From the best model structures found to fit with the experimental LEED $I(V)$ data, the Mo(112) surface relaxation is characterized by a surface contraction. There is a significant contraction $(-14.9 \%)$ of the spacing between the first layer and the second layer, accompanied by a shift $(0.05 \AA)$ of the surface atomic rows along the [11i] direction towards higher coordination positions. Although this shift is not so large, it allows the surface Mo atoms to move closer to subsurface atoms, thus decreasing interlayer spacing. One should note that for LEED taken at normal incidence there is a loss in sensitivity to the lateral movement in the $x-y$ plane, a complication that we do not fully address here.

As an independent check of the surface layer contraction of the $\mathrm{Mo}(112)$ surface, as determined by fittings to the experimental LEED $I(V)$ analysis, the stability of the Mo(112) surface was also analyzed by a comparison of surface energies obtained by first-principle calculations using the projected augmented-wave (PAW) method implemented in the Vienna $a b$ initio simulation package (VASP) [49]. The exchangecorrelation potential was treated in the generalized gradient approximation (GGA). We used an energy cut-off of $350 \mathrm{eV}$ for the plane wave expansion of the projected augmentedwaves and a $(10 \times 10 \times 1)$ Monkhorst-Pack grid for $k$ point sampling. Other details of the calculations can be found elsewhere $[49,50]$. All the structural relaxations were performed until the Hellman-Feynman forces on the relaxed atoms become less than $1 \mathrm{meV} \AA^{-1}$. In our optimization of surface energy, the slab was composed of 18 layers of the body-centered cubic structure of Mo(112) surfaces. Since the (112) surface has a periodicity of six layers, consistent with our LEED $I(V)$ analysis, the middle six layers served as the bulk and the top and bottom six layers are used to simulate the surface region. The possible geometrical relaxations must be constrained by the mirror plane symmetries. The initial lattice constant was chosen to be $3.15 \AA$, which corresponds to a bulk layer spacing of $1.28 \AA$. During optimization, the topmost six layers are treated as a single composite layer (surface), in which all the atomic positions are varied. These include vertical and lateral parameters of each atom. Deeper layers within the slab were kept at the values of their bulk positions.

From both LEED $I(V)$ analysis and surface energy optimization, the most remarkable result is the multilayer relaxation in the $z$ direction, i.e. along the surface normal. From table 1, we can see that the first layer contraction is consistent with previous studies [46, 47]. The Mo atoms in the deeper (subsurface) layers are also involved into the surface relaxation. Although difference exists among different studies, this disagreement does not exceed the error. Overall, there is a general consensus as to the surface relaxation of $\mathrm{Mo}(112)$, although there are some variations in the details of the surface structure. The Mo(112) surface shows oscillation behavior (contraction and expansion), alternating in sign for the $z$ direction ([211] direction). The mechanism of the multilayer relaxation near the surface was provided by Cho [51] and in their model, the oscillatory relaxation of the layers within the surface region is driven by the Friedel oscillations in the electron density. The period of the surface relaxation oscillations, which appears as a variation from the bulk in the 


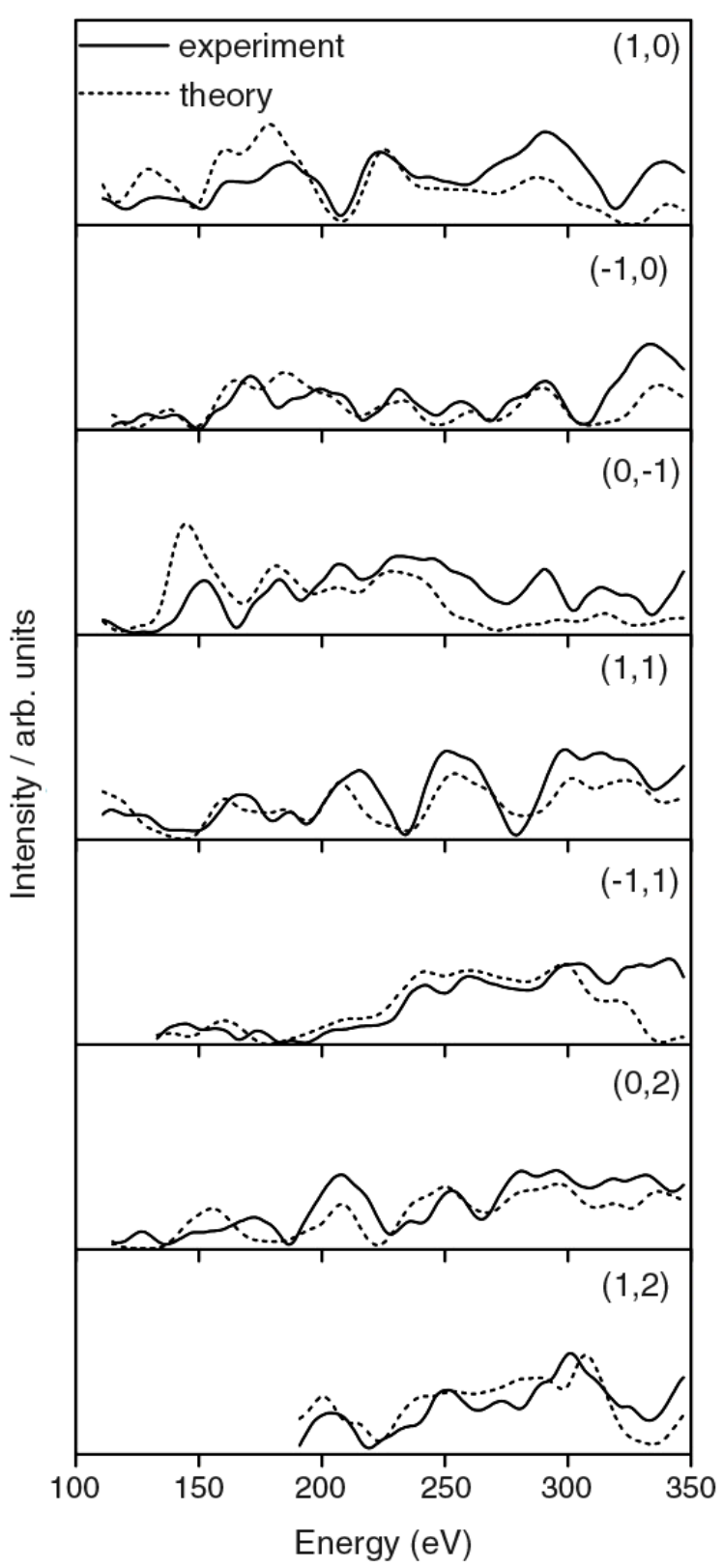

Figure 2. Comparison of the measured LEED $I(V)$ intensities for selected diffraction beams (solid lines) and calculated (dashed lines) fittings for the best-fit structure of the Mo(112) surface.

layer by layer spacing, follows the wavelength of the Friedel oscillation rather than the periodicity of the lattice. This may explain why in our analysis of LEED $I(V)$ data, the $\Delta d_{23}$ and $\Delta d_{34}$ lattice changes are smaller and $\Delta d_{45}$ and $\Delta d_{56}$ spacings are larger.

The surface relaxation for $\mathrm{Mo}(112)$ is significantly larger than is observed for the more close packed surface of Mo(100). This is summarized in table 1. At first glance this might just be the expected outcome for a more open surface like Mo(112) when compared to a more close packed surface like the (100) surface. The question as to whether the extent of the surface relaxation is simply related to the density (close packed nature) of the surface is perhaps more complex than appears from the comparison of the layer spacing data provided for molybdenum

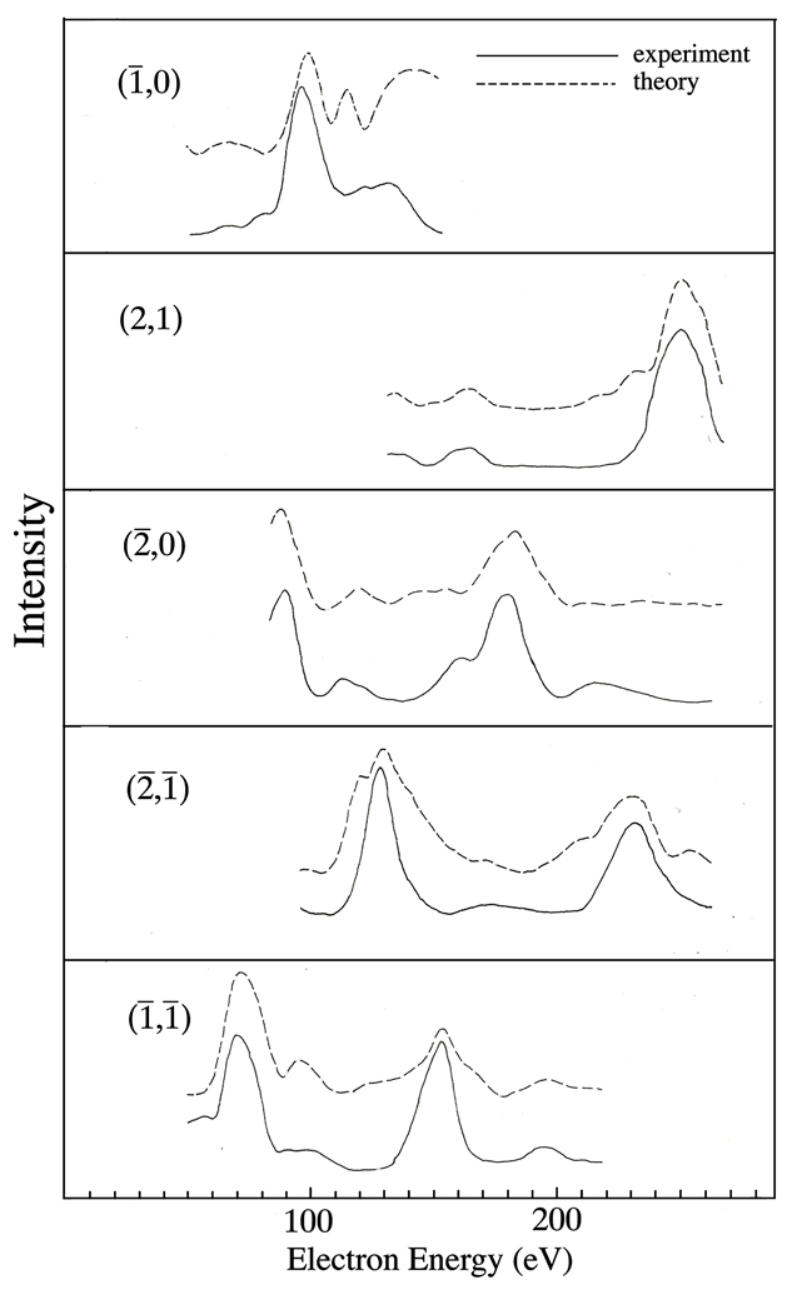

Figure 3. Comparison of measured LEED $I(V)$ intensities for selected diffraction beams (solid lines) with model dynamical scattering predictions (dashed lines) for the best-fit structure for the $\mathrm{Fe}(100)$ surface.

surfaces alone. We urge caution in applying the argument that the more open surface should exhibit the greater surface structural relaxation for the bcc metals. To illustrate why such caution is necessary, we can compare the surface relaxation parameters for the (112) and (100) surfaces for another bcc metal, in this case iron.

In relating the surface relaxation to the atomic surface density and surface orientation for another bcc metal (iron) a somewhat similar picture emerges, as summarized in table 1, but with significant differences. To compare the details of the (112) and (100) surfaces for the bcc metal iron, a re-analysis of the surface layer contraction for the $\mathrm{Fe}(100)$ surface has been included, based on LEED $I(V)$ data taken for the clean $\mathrm{Fe}(100)$ surface. The comparison of unpublished experimental LEED $I(V)$ data for $\mathrm{Fe}(100)$ and theoretical best-fit LEED $I(V)$ curves of five different beams of the $\mathrm{Fe}(100)$ surface is shown in figure 3. Our study indicates a contraction of the first $\mathrm{Fe}-\mathrm{Fe}$ layer of $10 \pm 2 \%$ that is substantially larger than the $0-3 \%$ estimate for the $\mathrm{Fe}(100)$ surface contraction obtained by Legg et al [56]. This new estimate of the surface layer contraction for $\mathrm{Fe}(100)$ comes from a fitting to the 
Table 1. Summaries of the experimental and theoretical data for the interlayer relaxation at bcc (112) and (100) surfaces of Mo and Fe. The surface interlayer relaxation values are in terms of the per cent of the bulk lattice spacing. Each value $\Delta d_{i j}$ is the relaxation of the interlayer spacing between layer $i$ and layer $j$, with respect to their respective bulk values provided in the per cent change calculated or estimated. Calculations indicated LDA were performed by the local density approximation. LDF indicates calculations done by the local density functional formalism, and in the case of [17] this is a first principles calculation within the local density functional formalism with the Hedin-Lundquist form of exchange and correlation. GTP indicates calculations done using the generalized pseudo-potential theory. PAW indicates calculations done by the projected augmented-wave methods (VASP). Throughout $(*)$ indicates this work.

\begin{tabular}{|c|c|c|c|c|c|c|}
\hline Surface & Source & $\Delta d_{12}$ & $\Delta d_{23}$ & $\Delta d_{34}$ & $\Delta d_{45}$ & $\Delta d_{56}$ \\
\hline \multicolumn{7}{|l|}{$\operatorname{Mo}(112)$} \\
\hline \multicolumn{7}{|l|}{ Experiment } \\
\hline & LEED* & -14.9 & 0.86 & -0.4 & 3.9 & -2.34 \\
\hline & LEED [45] & $-16 \pm 2$ & $2 \pm 3$ & $-2 \pm 3$ & $0 \pm 4$ & \\
\hline \multicolumn{7}{|l|}{ Theory } \\
\hline & PAW* & -17.6 & 2.64 & -1.2 & 3.4 & -2.02 \\
\hline & LDA [46] & -14 & -0.4 & & & \\
\hline & LDF [47] & -16.2 & 2.9 & -1.9 & 2.2 & -1.8 \\
\hline \multicolumn{7}{|l|}{$\operatorname{Mo}(100)$} \\
\hline \multicolumn{7}{|l|}{ Experiment } \\
\hline & LEED [52] & $-11.5 \%$ & & & & \\
\hline & LEED [53] & $-9.5 \pm 3$ & $1.0 \pm 2$ & & & \\
\hline \multicolumn{7}{|l|}{ Theory } \\
\hline & $\begin{array}{l}\text { LDF [17] } \\
\text { FP-LMTO [54] }\end{array}$ & $\begin{array}{l}-10.7 \\
-9\end{array}$ & 2.7 & 0.3 & & \\
\hline & LDF [47] & -11.1 & 2.3 & -1.7 & 0.3 & -0.6 \\
\hline & GPT [55] & -10.2 & 1.3 & & & \\
\hline \multicolumn{7}{|l|}{$\mathrm{Fe}(100)$} \\
\hline \multicolumn{7}{|l|}{ Experiment } \\
\hline & LEED* & $-10 \%$ & & & & \\
\hline & LEED [56] & $-1.4 \pm 3$ & & & & \\
\hline \multicolumn{7}{|l|}{$\mathrm{Fe}(112)$} \\
\hline \multicolumn{7}{|l|}{ Experiment } \\
\hline & LEED $[57,58]$ & $-10.4 \pm 2.6$ & $5.4 \pm 2.6$ & $-1.3 \pm 3.4$ & & \\
\hline & LEED [59] & $-11 \pm 1$ & $5 \pm 1$ & $-4 \pm 1$ & & \\
\hline Ineory & Point ion model [60] & -10.6 & 4.1 & -1.6 & 0.6 & -0.2 \\
\hline
\end{tabular}

LEED $I(V)$ data following the procedure of Clarke [53, 57], with an extremely good $R$-factor fitting, as summarized in figure 4. Indeed, our surface layer relaxation for $\mathrm{Fe}(100)$ does resemble the surface relaxation of the $\mathrm{Fe}(112)$ surface [58-61], as summarized in table 1. Thus for another bcc metal, in this case iron, the differences between the (100) and (112) surfaces are perhaps not as profound as the differences observed in the case of $\mathrm{Mo}(112)$ and $\mathrm{Mo}(100)$. The choice of inner potential may strongly affect the minimum of the $R$-factor and therefore the estimated surface layer contraction. Since there is always some uncertainty in the inner potential value, comparisons were done for a whole series of inner potentials for the $\mathrm{Fe}(100)$ fitting to the LEED $I(V)$ data, as plotted against surface layer contraction in figure 4.

What is clear is that the $\mathrm{Fe}(112)$ surface shows the characteristic contraction, expansion, and contraction of $\Delta d_{12}, \Delta d_{23}$, and $\Delta d_{34}$ layer spacings seen with the $\operatorname{Mo}(112)$ surface. The lesson here is that the surface exhibits significant surface layer relaxations, even in the absence of lateral reconstructions. Furthermore, the expectations concerning the exact surface structure that one might infer for one type of lattice (say a transition metal bcc surface), no matter how similar to another metal surface, should only be applied with great care. The superficial comparison provided here for the (112) and (100) surfaces of iron and molybdenum suggests that a more complicated picture may apply than simply the

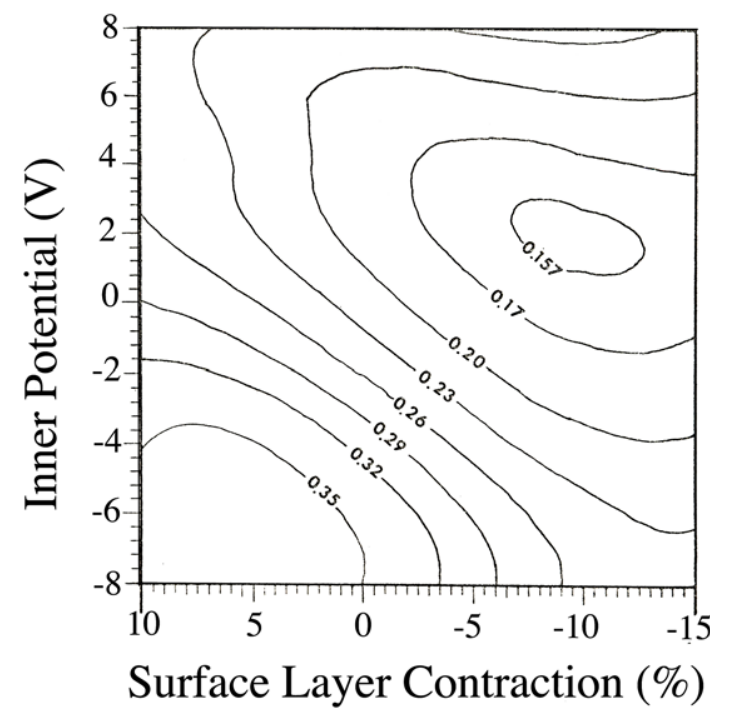

Figure 4. Comparison of $I(E)$ curves plotted as contours of overall reliability factors ( $R$-factor) against inner potential and surface relaxation for the $\mathrm{Fe}(100)$ surface, based on dynamical scattering analysis of the LEED $I(V)$ data for multiple diffraction beams.

density of atoms at the bcc lattice surface. Without the correct surface structure, the calculated surface band structure could be flawed. 


\section{The effective surface Debye temperature}

While not providing direct insight into the quasi-particle band structure, one of the easiest demonstrations of electron-phonon coupling in electron spectroscopy is the measurement of the effective surface Debye temperature. The true surface Debye temperature, containing the in-plane and anharmonic motions, is difficult to measure in most surface spectroscopies [29, 57], but the effective Debye temperature characteristic of motion along the surface normal can be readily obtained using LEED, x-ray photoemission (XPS), electron energy loss spectroscopy (EELS), inverse photoemission spectroscopy (IPES), and other surface sensitive techniques [29, 57, 62-68].

The dynamic motion of surface atoms, nonetheless, is a contribution to the scattering processes in experimental techniques such as LEED, photoemission, atomic beam scattering, low energy and medium energy ion beam scattering, and $\mathrm{x}$-ray scattering. Some of the apparent inconsistencies in the derived effective Debye temperature could arise in the analysis of the data obtained by the different techniques. The photoemission taken with the photoelectrons collected at normal emission only takes account of the perpendicular atomic motion, while there is some inclusion and averaging of the parallel and perpendicular motion in the measurements taken using LEED. The problem of anisotropic motion for some surfaces makes the comparison of different techniques even harder.

Generally, since the intensity of an emitted or scattered electron beam exponentially decays, with increasing temperature, due to increases in the thermal vibration, we can calculate the effective Debye temperature with careful analysis of the intensity change as a function of temperature [29, 57, 62]:

$$
\begin{gathered}
I=I_{0} \exp (-2 W) \\
2 W=\frac{3 \hbar^{2} T(\Delta k)^{2}}{2 m k_{\mathrm{B}} \Theta_{\mathrm{D}}^{2}}
\end{gathered}
$$

where $W$ is the Debye-Waller factor, $T$ is the temperature of the sample (in kelvin), $\hbar(\Delta k)$ is the electron momentum transfer, $m$ is the mass of the scattering center, $k_{\mathrm{B}}$ is the Boltzmann constant, and $\Theta_{\mathrm{D}}$ is the Debye temperature. Because of the different scattering geometries, we estimate the electron momentum transfer differently for XPS and LEED. For LEED, the momentum transfer is

$$
|\Delta \vec{k}|=\left|\vec{k}_{f}-\vec{k}_{i}\right|=\left|2 k \cos \frac{\theta}{2}\right|
$$

where $\theta$ is the angle between the surface normal and diffracted electron beam. Due to the geometry of LEED, $\theta$ is very small and $\sin ^{2} \frac{\theta}{2}$ (parallel component) is much smaller than $\cos ^{2} \frac{\theta}{2}$ (normal component). As noted at the outset, the calculated effective surface Debye temperature is most indicative of the dynamic motion of vibrational modes normal to the surface [29], hence the distinction from the true Debye temperature per se.

The effective Debye temperature of $313 \mathrm{~K}$ for the Mo(112) surface obtained using photoemission [29], does compare reasonably well with the value of $345 \mathrm{~K}$ obtained from the dynamical scattering analysis of the LEED $I(V)$ data, as described in the previous section. These values for the effective surface Debye temperature of $\mathrm{Mo}(112)$ are actually significantly higher than the estimates for the effective surface Debye temperature of $\mathrm{Mo}(100)$ of $230 \mathrm{~K}$ [53], but well below the bulk Debye temperatures of $422 \pm 4 \mathrm{~K}$ measured by photoemission [29], and $380 \mathrm{~K}$ measured by x-ray scattering [69]. Indeed the more open surface of molybdenum, Mo(112) does not appear to have the lower effective surface Debye temperature.

Due to the fact that the atoms at the surface have fewer nearest neighbors than in the bulk, the vibrational motions of those surface atoms should be enhanced and thus the surface Debye temperature normally follows the $\sqrt{2}$ law. This simple $\sqrt{2}$ relationship model appear to 'fit' the $\operatorname{Mo}(100)$ surface far better than for the Mo(112) surface. The Mo(112) surface has an apparently higher effective surface Debye temperature than $\operatorname{Mo}(100)$, but it is the $\operatorname{Mo}(112)$ surface where the surface atoms have fewer closely spaced adjacent nearest neighbor atoms. However, because of difficulties in comparing different scattering techniques discussed above, there is no simple consensus for the ratio of effective surface Debye temperature to bulk Debye temperature.

In general, the frozen phonon total energy calculation [70] indicates that the energy gain from surface relaxation competes with the energies available from lateral surface reconstructions. The phonon instability caused by the strong coupling of the surface states at the Fermi level with lateral displacements via the longitudinal phonon mode $[17,71]$ will have a strong influence on some molybdenum and tungsten surfaces and, as noted above, these modes can be anharmonic. A more correct Debye temperature that includes in-plane movement and electron-phonon coupling is essential to explain the surface relaxation and surface reconstruction [22].

\section{The band structure of $\operatorname{Mo}(112)$}

There has already been much published on the experimental band structure of the $\mathrm{Mo}(112)$ surface. A fairly complete experimental band structure of the $\operatorname{Mo}(112)$ surface can be constructed by combining published data [30-32] with additional new data that includes both the occupied and unoccupied experimental band structure obtained from photoemission and inverse photoemission studies of the Mo(112) surface. This experimental band structure for both the occupied and unoccupied bands along $\bar{\Gamma}-\bar{X}$ (left panel) and $\bar{\Gamma}-\bar{Y}$ (right panel) directions of the surface Brillouin zone is shown in figure 5, using the common notation for the Mo(112) Brillouin zone critical points and directions shown in the schematic diagram in figure 1. The experimental band structure is in general agreement with prior band structure calculations $[31,46]$, but we have revisited the calculated band structure of $\operatorname{Mo}(112)$ to include all of the known experimentally determined corrections for the surface structure of $\operatorname{Mo}(112)$.

The calculated band structure is plotted in figure 6 , with the bands with strong surface weight indicated in color (blue lines indicate those states with strong surface weight). There 


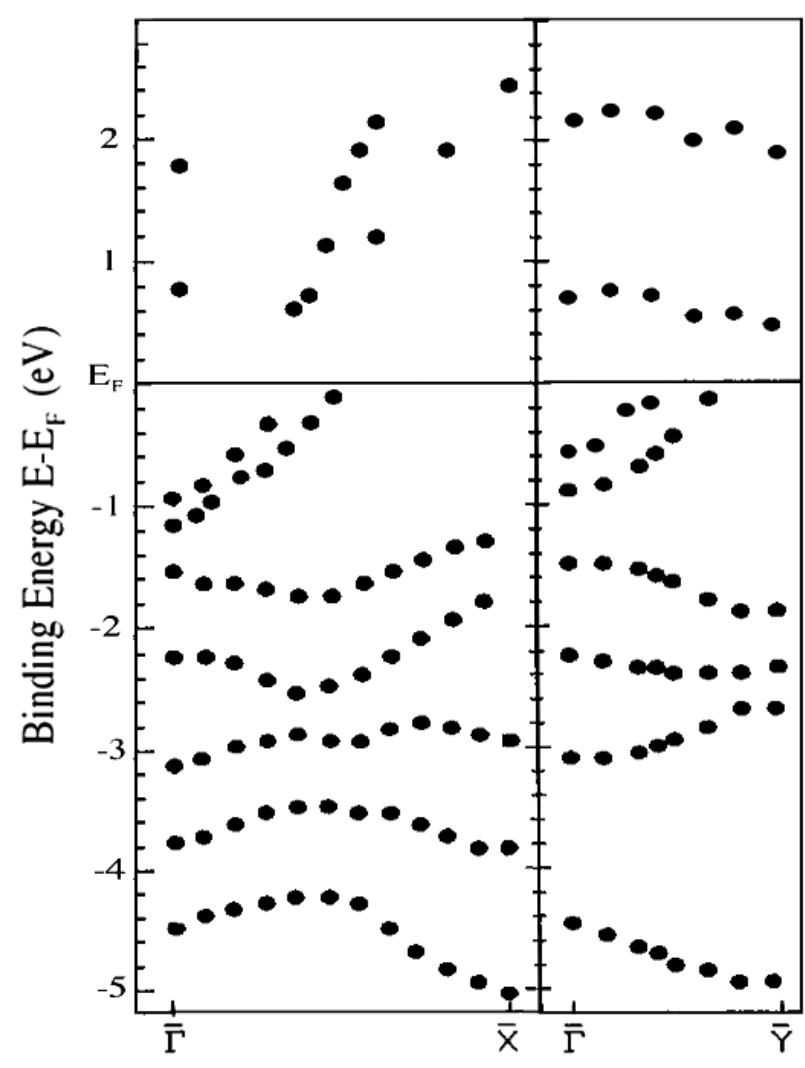

Figure 5. The experimental band structure for Mo(112) compiled from photoemission and inverse photoemission spectra taken largely from [30-32]. The band structures along $\bar{\Gamma}-\overline{\mathrm{X}}$ and $\bar{\Gamma}-\overline{\mathrm{Y}}$ are shown.

is agreement between the experimental surface resonance crossing $E_{\mathrm{F}}$ at about midway along $\bar{\Gamma}-\overline{\mathrm{X}}$ and a calculated surface resonance/surface state with Fermi level crossing at about the same position along $\bar{\Gamma}-\overline{\mathrm{X}}[31]$.

When the measured photoemission binding energies do not change with photon energy (no dependence upon the wavevector normal to the surface $k_{\perp}$ ), this indicates conservation of two dimensionality of state as well as both strong surface weight and likely surface sensitivity. On the photoemission side, a doublet of states, possibly caused by spin-orbital splitting, appears to dip across into the unoccupied bands at about $0.45-0.55 \AA^{-1}$ as these bands approach the Fermi level $\bar{\Gamma}-\overline{\mathrm{X}}[30,31]$. The emergence of the band above $E_{\mathrm{F}}$, as seen with inverse photoemission, confirms that there is a Fermi level crossing in this region of the Brillouin zone at about $0.5 \AA^{-1}$. The band crossing of the Fermi level at about midway along $\bar{\Gamma}-\bar{X}$ (located at a binding energy in the region of $-1.2 \mathrm{eV}$ at $\bar{\Gamma}$ ) is largely unaffected by photon energy, as one approaches the Fermi level crossing, consistent with strong surface weight $[30,31]$. This assignment of surface weight to the band in the region of the Fermi level band crossing at $0.54 \AA^{-1}$ is consistent with the calculations summarized in figure 6. The assignment of surface weight to the state where there is a crossing of the Fermi level in the region of $0.54 \AA^{-1}$ is also indicated by the sensitivity of this state to contamination [30]. Another band located $1 \mathrm{eV}$ below the Fermi level (at $\bar{\Gamma}$ ) (with a binding energy $E-E_{\mathrm{F}}$ ranging

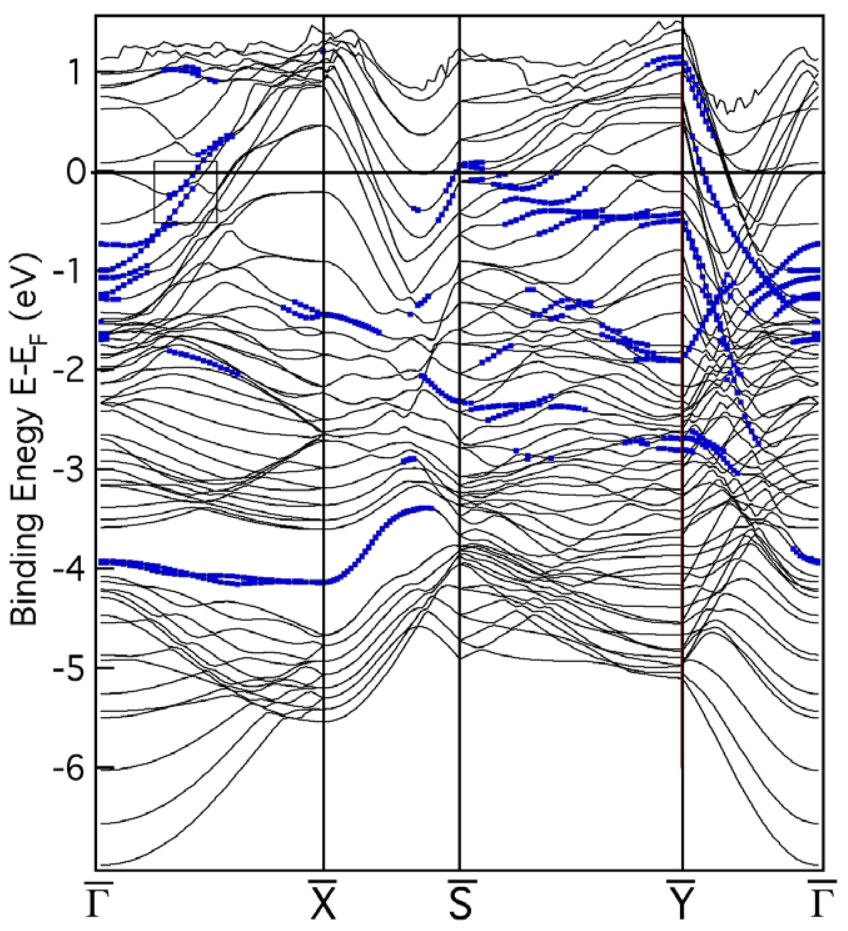

Figure 6. The theoretical band structure for $\operatorname{Mo}(112)$ is plotted where blue lines represent the bands with strong surface weight either as surface bands or surface resonance. A box in the calculated band structures along $\bar{\Gamma}$ to $\overline{\mathrm{X}}$ indicates the region of interest where mass enhancement due to the electron-phonon has been experimentally identified (see text and figure 7).

from -0.3 to $-1 \mathrm{eV}$ ) has a dispersion that varies with photon energy and this dependence on the wavevector $k_{\perp}$ normal to the surface is indicative of a band with bulk band structure contributions [30, 31].

The calculated band structure (figure 6) does not, however, include the electron-phonon coupling which can significantly alter the quasi-particle mass in a narrow energy region close to the Fermi level. This $k_{\|}$dependent quasi-particle band structure for the strongly surface weighted band crossing the Fermi level in the region of $0.54 \AA^{-1}$ along $\bar{\Gamma}-\bar{X}$ (figures 5 and 6 ) is evident in the much higher resolution photoemission spectrum shown in figure 7.

To better illustrate the increase in the effective mass due to the electron-phonon coupling in this region of the Fermi level, only a small region of the surface Brillouin zone from 0.26 to $0.61 \AA^{-1}$ has been plotted out. With the high energy and wavevector resolution, the electron-phonon coupling induced mass enhancement of the band near the Fermi level in the region of $0.54 \AA^{-1}$ along $\bar{\Gamma}-\bar{X}$ of the surface Brillouin zone can be readily identified. This mass enhancement is clearly significant and more evident than that reported for the Mo(110) surface [3].

This surface resonance state crosses the Fermi level at around $0.54 \AA^{-1}$, a value for the Fermi level crossing that might be considered different from the range of possible values of 0.45 to $0.55 \AA^{-1}$ in figure 5. These differences in the wavevector of the Fermi level crossing are a result of the finite resolution of the data used to plot the experimental band 


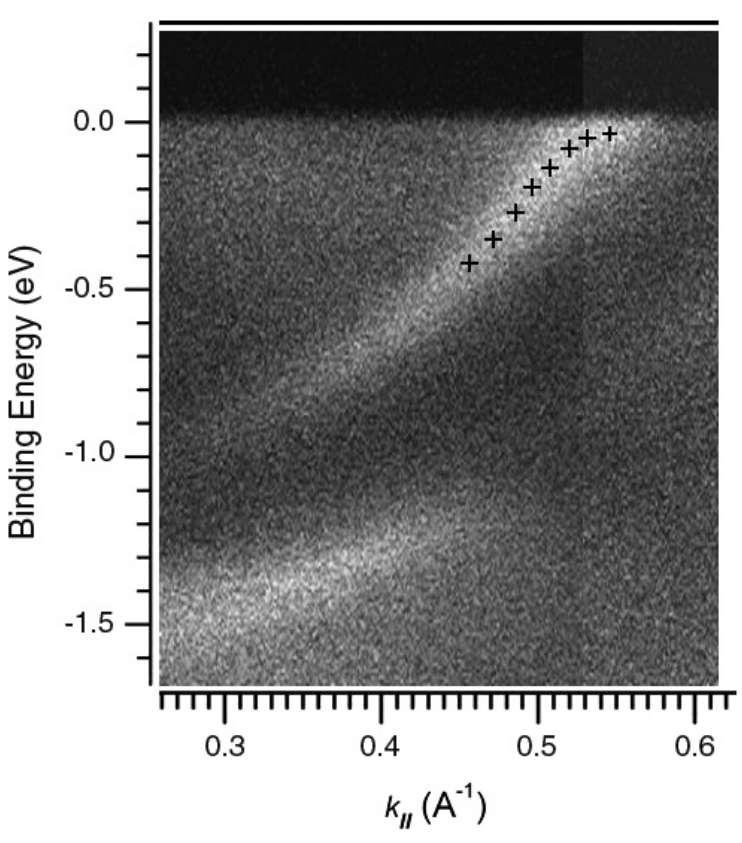

Figure 7. The high resolution band mapping over a limited range of wavevectors from 0.26 to $0.61 \AA^{-1}$ along the $\bar{\Gamma}$ to $\overline{\mathrm{X}}$ direction in the surface Brillouin zone taken at a photon energy of $18 \mathrm{eV}$. The mass enhancement due to the electron-phonon coupling in the region of the Fermi level is roughly indicated.

structure in figure 5 that fail to accurately depict the widths and position of the quasi-particle peak in photoemission, as a function of wavevector.

\section{Summary}

LEED $I(V)$ analysis and surface energy optimization were used to investigate the surface relaxation of $\mathrm{Mo}(112)$ surfaces. The oscillatory behavior of interlayer relaxation and contraction at the surface and near surface region of $\mathrm{Mo}(112)$ was found. Comparisons of the surface structure, in particular the percentage of surface relaxation for $\mathrm{Mo}(112)$ and $\mathrm{Mo}(100)$ with another bcc surface, in this case $\mathrm{Fe}(112)$ and $\mathrm{Fe}(100)$, suggest that while there are some similarities in the types of surface relaxations observed with both bcc metals, one should be cautious to not over generalize the influence of surface structure on the basis of the surface crystal face alone.

The electron band structure of $\mathrm{Mo}(112)$ along two directions in the surface Brillouin zone does exhibit bands with strong surface weight: either as surface states or surface resonances. One such band with strong surface weight exhibits electron-phonon coupling in the region of the Fermi level. This $k_{\|}$dependent quasi-particle band structure leads to a significant mass enhancement for the strongly surface weighted band crossing Fermi level in the region of $0.54 \AA^{-1}$ along the $\bar{\Gamma}-$ $\overline{\mathrm{X}}$ direction.

Obviously, there is now a large body of evidence that electron-phonon coupling can affect the band structure, particularly in the region of the Fermi level [1-3]. The resulting mass enhancement factors have not only now been characterized for a whole host of metal surfaces [1], but also for the surfaces of a semimetal like graphite [72] and the surface of a superconductor like $2 \mathrm{H}-\mathrm{NbS}_{2}$ [73]. As noted at the outset of this article, there is a strong interplay between the shifts in the surface band structure due to electronphonon coupling and the surface phonon spectra. In looking beyond this discussion of electron-phonon coupling in a paramagnetic metallic system such as molybdenum, electron wavevector $k_{\|}$dependent phonon coupling has been long predicted to be extremely important in magnetic systems [75]. We should expect, not ignore, a wavevector $k_{\|}$dependent quasi-particle band structure and a $k_{\|}$dependent Eliashberg function $[1,74,75]$.

\section{Acknowledgments}

The authors at the University of Nebraska were supported by Nebraska Research Initiative, the UNL NSF 'QSPINS' MRSEC (DMR-0820521). The J Bennett Johnston Sr. Center for Advanced Microstructures and Devices is supported by the Louisiana Board of Regents. Lionel Clarke is thanked for performing the dynamical LEED scattering analysis of the $\mathrm{Fe}(100) I(V)$ data reported here, and the authors would like to acknowledge a number of helpful discussions with John Inglesfield and Ivan Yakovkin.

\section{References}

[1] Plummer E W, Shi J, Tang S-J, Rotenberg E and Kevan S D 2003 Prog. Surf. Sci. 74251

[2] Nolting W 2009 Fundamentals of Many-Body Physics: Principles and Methods (Berlin: Springer) ISBN 978-3-540-71930-4

[3] Valla T, Fedorov A V, Johnson P D and Hulbert S L 1999 Phys. Rev. Lett. 832085

[4] Tamm I E 1932 Phys. Z. Sov. Union 1732

[5] Maue A-W 1935 Z. Phys. 94717

[6] Shockley W 1939 Phys. Rev. 56317

[7] Goodwin E T 1939 Proc. Camb. Phil. Soc. 35205

[8] Goodwin E T 1939 Proc. Camb. Phil. Soc. 35221

[9] Goodwin E T 1939 Proc. Camb. Phil. Soc. 35232

[10] Swanson L W and Crouser L C 1966 Phys. Rev. Lett. 16389

[11] Weng S-L, Gustafsson T and Plummer E W 1977 Phys. Rev. Lett. 39822

[12] Weng S-L, Gustafsson T and Plummer E W 1978 Phys. Rev. B 181718

[13] Felter T E, Barker R A and Estrup P J 1977 Phys. Rev. Lett. 381138

[14] Debe M K and King D A 1977 Phys. Rev. Lett. 39708

[15] Chung J W, Shin K S, Baek D H, Kim C Y, Kim H W, Lee S K, Park C Y, Kinoshita T, Watanabe M, Kakizaki A and Ishii T 1992 Phys. Rev. Lett. 692228

[16] Smith K E and Kevan S D 1991 Phys. Rev. B 433986

[17] Wang X W, Chan C T, Ho K M and Weber W 1988 Phys. Rev. Lett. 602066

[18] Terakura I, Terakura K and Hamada N 1981 Surf. Sci. 103103

[19] Barker R A, Estrup P J, Jona F and Marcus P M 1978 Solid State Commun. 25375

[20] Hulpke E and Smilgies D M 1989 Phys. Rev. B 101338

[21] Inglesfield J E 1978 J. Phys. C: Solid State Phys. 11 L69

[22] Inglesfield J E 1979 J. Phys. C: Solid State Phys. 12149

[23] Peierls R E 1955 Qunatum Theory of Solids (Oxford: Oxford University Press)

[24] Tosatti E 1978 Solid State Commun. 25637 
[25] Krakauer H, Posternak M and Freeman A J 1979 Phys. Rev. Lett. 431885

[26] Campuzano J C, King D A, Somerton C and Inglesfield J E 1980 Phys. Rev. Lett. 451649

[27] Campuzano J C, Inglesfield J E, King D A and Somerton C 1981 J. Phys. C: Solid State Phys. 143099

[28] Holmes M I and Gustafsson T 1981 Phys. Rev. Lett. 47443

[29] Waldfried C, McIloy D N, Zhang J, Dowben P A, Katrich G A and Plummer E W 1996 Surf. Sci. 363296

[30] McAvoy T, Zhang J, Waldfried C, McIlroy D N, Dowben P A, Zeybek O, Bertrams T and Barrett S D 2000 Eur. Phys. J. B $14747-55$

[31] Yakovkin I N, Zhang J and Dowben P A 2001 Phys. Rev. B 63115408

[32] Jeong H-K, Komesu T, Yakovkin I N and Dowben P A 2001 Surf. Sci. Lett. 494 L773-80

[33] Schroeder T, Zegenhagen J, Magg N, Immaraporn B and Freund F J 2004 Surf. Sci. $\mathbf{5 5 2} 85$

[34] Kaya S, Weissenrieder J, Stacchiola D, Todorova T K, Sierka M, Sauer J, Shaikhutdinov S and Freund H J 2008 Surf. Sci. $\mathbf{6 0 2} 3338$

[35] Yakovkin I N, Kuchowicz M, Szukiewicz R and Kolaczkiewicz J 2006 Surf. Sci. 600 L240

[36] Kuchowicz M, Stepanovsky S and Kolaczkiewicz J 2006 Surf. Sci. 6001600

[37] Fukui K, Aruga T and Iwasawa Y 1993 Surf. Sci. 281241

[38] Sasaki T, Goto Y, Tero R, Fukui K and Iwasawa Y 2002 Surf. Sci. $\mathbf{5 0 2} 136$

[39] Schroeder T, Giorgi J B, Hammoudeh A, Magg N, Baumer M and Freund H J 2002 Phys. Rev. B 65115411

[40] Losovyj Y, Ketsman I, Morikawa E, Wang Z, Tang J and Dowben P 2007 Nucl. Instrum. Methods Phys. Res. A 582264

[41] Dowben P A, LaGraffe D and Onellion M 1989 J. Phys.: Condens. Matter 16571

[42] Hormes J, Scott J D and Suller V P 2006 Synchrotron Radiat. News 1927

[43] Losovyj Y, Morris K, Rosa L, Scott J D and Dowben P 2007 Nucl. Instrum. Methods Phys. Res. A 582258

[44] Evans C M, Scott J D and Morikawa E 2002 Rev. Sci. Instrum. 731557

[45] Kolthoff D, Pfur H, Fedorus A G, Koval V and Naumovets A G 1999 Surf. Sci. 439224

[46] Yakovkin I N 2005 Eur. Phys. J. B 44551

[47] Che J G, Chan C T, Jian W-E and Leung T C 1998 Phys. Rev. B 571875

[48] Rous P J, Pendry J B, Saldin D K, Heinz K, Muller K and Bickel N 1986 Phys. Rev. Lett. 572951

[49] Kresse G and Joubert D 1999 Phys. Rev. B 591758

[50] Sabiryanov R F, Larsson M I, Cho K J, Nix W D and Clemens B M 2003 Phys. Rev. B 67125412
[51] Cho J-H, Ismail, Zhang Z and Plummer E W 1999 Phys. Rev. B 591677

[52] Ignatiev A, Jona F, Shih H D, Jepsen D W and Marcus P M 1975 Phys. Rev. B 114787

[53] Clarke L J 1980 Surf. Sci. 91131

[54] Methfessel M, Hennig D and Scheffler M 1992 Phys. Rev. B 464816

[55] Moriarty J A and Phillips R 1991 Phys. Rev. Lett. 663036

[56] Legg K O, Jona F, Jepsen D W and Marcus P M 1977 J. Phys. C: Solid State Phys. 10937

[57] Clarke L J 1985 Surface Crystallography (New York: Wiley)

[58] Sokolov J, Jona F and Marcus P M 1984 Solid State Commun. 49307

[59] Sokolov J, Shih H D, Bardi U, Jona F and Marcus P M 1984 J. Phys. C: Solid State Phys. 2371

[60] Hassold E, Löffler U, Schmiedl R, Grund M, Hammer L, Heinz K and Müller K 1995 Surf. Sci. 32693

[61] Jiang P, Marcus P M and Jona F 1986 Solid State Commun. 59275

[62] Van Hove M A, Weinberg W H and Chan C M 1986 Low-Energy Electron Diffraction (Springer Series in Surface Science vol 6) (Berlin: Springer) p 134

[63] Borca C N, Ristoiu D, Komesu T, Jeong H-K, Hordequin Ch, Pierre J, Nozieres J P and Dowben P A 2000 Appl. Phys. Lett. 77 88-90

[64] Borca C N, Choi J, Adenwalla S, Ducharme S, Dowben P A, Robertson L, Fridkin V M, Palto S P and Petukhova N 1999 Appl. Phys. Lett. 74 347-9

[65] Borca C N, Xu B, Komesu T, Jeong H K, Liu M T, Liou S H and Dowben P A 2002 Sci. Lett. 512 L346

[66] Tonner B P, Li H, Robrecht M J, Chou Y C, Onellion M and Erskine J L 1986 Phys. Rev. B 344386

[67] Kevan S D and Shirley D A 1980 Phys. Rev. B 22542

[68] Williams R S, Wehner P S, Stöhr J and Shirley D A 1977 Phys. Rev. Lett. 39302

[69] James R W 1965 The Optical Principles of the Diffractions of $X$-rays (Ithaca, NY: Cornell University Press)

[70] Fu C L, Freeman A J and Wimmer E 1985 Phys. Rev. Lett. 542261

[71] Wang X W and Weber W 1987 Phys. Rev. Lett. 581452

[72] Leem C S, Kim C, Park S R, Kim M-K, Choi H J, Kim C, Kim B J, Johnson S, Devereaux T, Ohta T, Bostwick A and Rotenberg E 2009 Phys. Rev. B 79125438

[73] Valla T, Fedorov A V, Johnson P D, Glans P-A, McGuinness C, Smith K E, Andrei E Y and Berger H 2003 Phys. Rev. Lett. 92086401

[74] Grimvall G 1981 The Electron-Phonon Coupling in Metals (Selected Topics in Solid State Physics) ed E Wohlfarth (New York: North-Holland)

[75] Kim D J 1988 Phys. Rep. 171129 